\title{
Antidisturbance Fault Tolerant Control of Attitude Control Systems for Microsatellite with Unknown Input Delay
}

\author{
Jianzhong Qiao' ${ }^{1}$ and Lei Guo ${ }^{1,2}$ \\ ${ }^{1}$ School of Instrumentation Science and Opto-Electronics Engineering, Beihang University, Beijing 100191, China \\ ${ }^{2}$ Science and Technology on Aircraft Control Laboratory, Beihang University, Beijing 100191, China \\ Correspondence should be addressed to Jianzhong Qiao; qiaojianzhong83@163.com
}

Received 23 August 2013; Accepted 24 September 2013

Academic Editor: Tao Li

Copyright (C) 2013 J. Qiao and L. Guo. This is an open access article distributed under the Creative Commons Attribution License, which permits unrestricted use, distribution, and reproduction in any medium, provided the original work is properly cited.

\begin{abstract}
The antidisturbance fault tolerant control problem of attitude control systems for microsatellite is investigated in the presence of unknown input delay, stuck faults from the reaction wheel and the multiple disturbances. The multiple disturbances are supposed to include the vibration disturbance torque from the reaction wheel and modeling uncertainties. The fault diagnosis observer and disturbance observer are constructed to estimate stuck faults and vibration disturbance torque from the reaction wheel, respectively. A composite fault tolerant controller is designed by combining a PID controller, the fault accommodation estimation based on the fault diagnosis observer, and the disturbance compensator based on the disturbance observer. The controller and observer gains can be easily obtained via a set of linear matrix inequalities. Simulation results are given to show that the faults can be accommodated readily, and the disturbances can be rejected and attenuated simultaneously.
\end{abstract}

\section{Introduction}

Microsatellite, which is a kind of modern small satellites with mass below $100 \mathrm{~kg}$, plays more and more important roles in position location, earth observation, atmospheric data collection, space science, and other space missions because of its advantages of cost, risk, and manufacturing time. The reaction wheel had been widely used for microsatellite attitude control as a kind of momentum exchange device owing to its great decrease in size and weight. In order to pursue designated space missions, the reaction wheel works on a high-speed rotational state sometimes; it raises the probability of faults to occur inevitably (see $[1,2]$ ). Moreover, the wheel produces a disturbance torque and force as by products; the effects of wheel disturbances on the microsatellite's attitude error and stability are so critical that the influence of disturbances on the quality of the attitude control should be analyzed prior to the application of the wheels for microsatellite (see [3-6]). In addition, it is well known that time-delay is a common phenomenon in many industrial and engineering system and is one of the instability sources for dynamical systems (see [7-9]). With an impending requirement on reliability and stability of attitude control systems, time delay must be considered when designing the control algorithm. All these aspects in a realistic environment create considerable difficulty in the design of attitude control systems for adequate performance and stability, especially when all these issues are treated simultaneously.

In the past several decades, one way to improve the reliability is that enhancing the fault tolerance of the system. More and more attention had been paid to the development of methodologies to detect and isolate faults so that measures could be taken to accommodate these faults (see [10-13]). In [14], a simple and effective fault tolerant control method for satellites with four reaction wheels has been proposed based on dynamic inversion and time-delay control theory. In [15], a nonlinear fault detection and isolation strategy for redundant reaction wheels in the attitude control subsystem for a satellite has been attempted. However, the strategy for redundant reaction wheels is unfeasible for microsatellite because of its size and weight. 
To remedy this problem, more and more attention had been focused on model-based methodologies. In [16], an interactive bank of unscented Kalman filters has been developed for fault detection and isolation in reaction wheel actuators of satellite attitude control systems. In [17], an unsupervised algorithm of kernel fuzzy $c$-means-based fault diagnosis method for unknown faults in satellite reaction wheels has been presented. Because of continued presence of disturbance, the fault tolerant control (FTC) problem of attitude control systems for microsatellite with multiple disturbances will be more complicated.

To overcome these obstacles, some approaches have been provided and applied. In [18], a robust adaptive FTC approach for attitude tracking of flexible spacecraft is proposed for use in situations when there are reaction wheels/actuator failures, external disturbances, and time-varying inertia-parameter uncertainties. In [19], a FTC design technique against actuator stuck faults with application to spacecraft attitude maneuvering control systems has been proposed via designing an integral-type sliding mode attitude controller to compensate for the effects of stuck actuators and system parameters and external disturbances. In [20], a robust adaptive controller has been provided with the utilization of fuzzy logic and backstepping techniques. The authors investigate the problem of spacecraft in the presence of unknown mass moment of inertia matrix, external disturbances, actuator failures, and control input constraints. Overall, some robust control techniques had been applied in most existing literature for the disturbances are assumed to be norm bounded. However, in practical engineering, the disturbance may originate from various sources and can be described by a composite form rather than a single variable. In this case, the robust control may be too conservative to provide highly accurate control performance. As such, disturbance attenuation and rejection for linear (or nonlinear) control systems is a challenging objective in the control area. Analysis and synthesis for control systems with disturbances have been one of the most active research fields in the past few decades. The idea of disturbance-observer-based control (DOBC) is to construct an observer to estimate and compensate some external disturbances (see [21-27]). And it has been shown that DOBC approach has a good performance to reject the various unknown disturbances. However, none of these methods has dealt with multiple disturbances as well as unknown input delay simultaneously.

In this paper, a composite fault tolerant controller is firstly addressed for the attitude control systems for microsatellite with stuck faults from reaction wheel, multiple disturbances and unknown input delay based on fault diagnosis observer, disturbance observer and PID state-feedback controller. Fault diagnosis observer can estimate the stuck faults from reaction wheel real time. Disturbance observer can estimate the effect of vibration from the reaction wheel. PID state-feedback controller can attenuate the influence of the norm bounded disturbances and the estimation errors. Simulation results for a microsatellite are given to show the efficiency of the proposed approach.

The remainder of this paper is organized as follows. In Section 2, the dynamic model for microsatellite and control problem formulation is presented. In Section 3, the fault diagnosis observer and disturbance observer are designed. In Section 4, the stabilization of the attitude control systems under the given controller is analyzed, and the solution of the controller is resolved. In Section 5, the proposed control algorithm is confirmed by numerical simulation. Section 6 contains conclusions.

\section{Problem Formulation}

When the Euler angle is very small, the attitude dynamics equation for microsatellite can be described by the following matrix form:

$$
\begin{gathered}
I_{1} \ddot{\gamma}-\omega\left(I_{1}-I_{2}+I_{3}\right) \dot{\theta}+4 \omega^{2}\left(I_{2}-I_{3}\right) \gamma \\
=u_{1}(t-\Delta(t))+F_{1}+M_{d 1}, \\
I_{2} \ddot{\varphi}+3 \omega^{2}\left(I_{1}-I_{3}\right) \varphi=u_{2}(t-\Delta(t))+F_{2}+M_{d 2}, \\
I_{3} \ddot{\theta}+\omega\left(I_{1}-I_{2}+I_{3}\right) \dot{\gamma}+\omega^{2}\left(I_{2}-I_{1}\right) \theta \\
=u_{3}(t-\Delta(t))+F_{3}+M_{d 3},
\end{gathered}
$$

where $\gamma, \varphi$, and $\theta$ are the yaw angle, pitch angle, and rolling angle, respectively. $I_{i}(i=1,2,3)$ is the inertia matrix, and $\omega$ is the velocity of the orbital reference frame with respect to the inertial frame expressed in the body-fixed reference frame. $\Delta(t)$ is an unknown control input delay, and the control input torque is supposed to be $u_{i}(t-\Delta(t)) . \Delta(t)$ satisfies $0 \leq \Delta(t) \leq$ $\tau<\infty$ and $\dot{\Delta}(t) \leq \bar{d}<1 . M_{d i}$ and $F_{i}$ are the disturbance torques and fault input vector, respectively.

Since the microsatellite rarely performs a large angle maneuver, the linear model is reasonably accurate and acceptable to be used in attitude controller design. So, the attitude dynamics equation can be rewritten as follows:

$$
\begin{aligned}
& M \ddot{p}(t)+C \dot{p}(t)+K p(t) \\
&=B_{u}\left(u(t-\Delta(t))+F(t)+d_{1}(t)\right)+B_{w} d_{2}(t),
\end{aligned}
$$

where $p(t)=[\gamma, \varphi, \theta]^{T}$ is the state vector of Euler angles, $\dot{p}(t)$ is the Euler angular velocity, and $\ddot{p}(t)$ is the Euler angles acceleration. $F(t)$ is the stuck fault from reaction wheel, and it is supposed to be time varying, but its derivative is supposed to be bounded. $d_{1}(t)$ is the disturbance which represents the vibration disturbance torque from reaction wheel. $d_{2}(t)$ is the merged disturbance from space environmental disturbances, moment-of-inertia uncertainty, and noises from sensors and actuators. Considering

$$
\begin{gathered}
M=\left[\begin{array}{ccc}
I_{1} & 0 & 0 \\
0 & I_{2} & 0 \\
0 & 0 & I_{3}
\end{array}\right], \\
C=\left[\begin{array}{ccc}
0 & 0 & -\omega\left(I_{1}-I_{2}+I_{3}\right) \\
0 & 0 & 0 \\
\omega\left(I_{1}-I_{2}+I_{3}\right) & 0 & 0
\end{array}\right],
\end{gathered}
$$




$$
\begin{gathered}
K=\left[\begin{array}{ccc}
4 \omega^{2}\left(I_{2}-I_{3}\right) & 0 & 0 \\
0 & 3 \omega^{2}\left(I_{1}-I_{3}\right) & 0 \\
0 & 0 & -\omega^{2}\left(I_{1}-I_{2}\right)
\end{array}\right], \\
B_{u}=B_{w}=\left[\begin{array}{lll}
1 & 0 & 0 \\
0 & 1 & 0 \\
0 & 0 & 1
\end{array}\right] .
\end{gathered}
$$

Define $x(t)=\left[\int_{0}^{t} p(\tau) d \tau, p(t), \dot{p}(t)\right]^{T}$ and then transform the attitude dynamics equation into state space model, which can be written as

$$
\dot{x}(t)=A x(t)+B_{1}\left(u(t-\Delta(t))+F(t)+d_{1}(t)\right)+B_{2} d_{2}(t),
$$

where

$$
\begin{gathered}
A=\left[\begin{array}{cc}
0 & I \\
-M^{-1} K & -M^{-1} C
\end{array}\right], \\
B_{1}=\left[\begin{array}{c}
0 \\
M^{-1} B_{u}
\end{array}\right], \quad B_{2}=\left[\begin{array}{c}
0 \\
M^{-1} B_{w}
\end{array}\right] .
\end{gathered}
$$

The vibration disturbance torque $d_{1}(t)$ is supposed to be described by

$$
d_{1}(t)=\sum_{i=1}^{n} C_{i} f_{r w a}^{2} \sin \left(2 \pi h_{i} f_{r w a} t+\phi_{i}\right),
$$

where $n$ is the number of harmonics included in the model, $C_{i}$ is the amplitude of the $i$ th harmonic, $f_{\text {rwa }}$ is the wheel speed in $\mathrm{Hz}, h_{i}$ is the $i$ th harmonic number, and $\phi_{i}$ is a random phase (assumed to be uniform over $[0,2 \pi]$ ) (see $[5,6])$. However, it is impossible to obtain the information of $C_{i}$ and $\phi_{i}$ except $f_{\text {rwa }}$ when the reaction wheel works on a high-speed rotational state. The disturbance force or torque $d_{1}(t)$ can be rewritten as the following matrix form:

$$
\begin{gathered}
d_{1}(t)=V w(t), \\
\dot{w}(t)=W w(t)+B_{3} \delta(t),
\end{gathered}
$$

where $w(t)$ is the state variable, and $\delta(t)$ is the additional disturbance that results from the perturbations and uncertainties in the exogenous system. $W$ is the vibration frequency of reaction wheel, and $V$ and $B_{3}$ are the known parameter matrices of the exogenous system with proper dimension.

Assumption 1. $\left(A, B_{1}\right)$ is controllable; $\left(W, B_{1} V\right)$ is observable.

In the next section, the objective is to design fault diagnosis observer and disturbance observer, with which the fault can be accommodated, and the modeled disturbance can be rejected.

\section{Fault Diagnosis Observer and Disturbance Observer Design}

3.1. Fault Diagnosis Observer Design. Fault diagnosis needs to be accomplished in order to reconfigure system and improve its reliability. For this purpose, the following fault diagnosis observer is constructed to diagnose the stuck faults from the reaction wheel

$$
\begin{aligned}
\widehat{F}(t)= & \varepsilon(t)-K x(t), \\
\dot{\varepsilon}(t)= & K B_{1}(\varepsilon(t)-K x(t)) \\
& +K\left[A x(t)+B_{1} u(t-\Delta(t))+B_{1} \widehat{d}_{1}(t)\right],
\end{aligned}
$$

where $\varepsilon(t)$ is the auxiliary variable, and $\widehat{F}(t)$ is the estimation of $F(t) . K$ is the fault diagnosis observer gain to be determined later. $\widehat{d}_{1}(t)$ is the estimation of $d_{1}(t)$ which will be designed in next subsection.

3.2. Disturbance Observer Design. Disturbance observer is designed in this subsection in order to reject the modeled external disturbance, and the disturbance observer is formulated as

$$
\begin{gathered}
\widehat{d}_{1}(t)=V \widehat{w}(t), \\
\widehat{w}(t)=v(t)-L x(t), \\
\dot{v}(t)=\left(W+L B_{1} V\right)(v(t)-L x(t)) \\
+L\left[A x(t)+B_{1} u(t-\Delta(t))+B_{1} \widehat{F}(t)\right],
\end{gathered}
$$

where $v(t)$ is the auxiliary variable, and $\widehat{w}(t)$ is the estimation of $w(t) . L$ is the disturbance observer gain to be determined later.

The error of disturbance observer and fault diagnosis observer are defined as

$$
\begin{aligned}
& e_{w}(t)=w(t)-\widehat{w}(t-\Delta(t)), \\
& e_{F}(t)=F(t)-\widehat{F}(t-\Delta(t))
\end{aligned}
$$

Then

$$
\begin{aligned}
\dot{e}(t)= & W_{1} e(t)+N B_{1} E e(t-\Delta(t)) \\
& +N B_{2} d_{2}(t-\Delta(t))+H_{3} \delta(t)+H_{1} \dot{F}(t),
\end{aligned}
$$

where

$$
\begin{aligned}
& e(t)=\left[\begin{array}{l}
e_{w}(t) \\
e_{F}(t)
\end{array}\right], \quad N=\left[\begin{array}{l}
L \\
K
\end{array}\right], \quad E=\left[\begin{array}{ll}
V & I
\end{array}\right], \\
& H_{3}=\left[\begin{array}{c}
B_{3} \\
0
\end{array}\right], \quad H_{1}=\left[\begin{array}{l}
0 \\
1
\end{array}\right], \quad W_{1}=\left[\begin{array}{cc}
W & 0 \\
0 & 0
\end{array}\right] .
\end{aligned}
$$

In this section, fault diagnosis observer and disturbance observer are designed for fault estimation and disturbance estimation, respectively. In the next section, a composite time-delay fault tolerant controller should be determined for reconfiguring the systems with disturbance rejection and attenuation performance.

\section{Composite Fault Tolerant Controller}

In this section, a composite fault tolerant controller is designed to guarantee system (7) stability in the presence of 
stuck faults and disturbances simultaneously. A composite fault tolerant controller is presented as

$$
u(t)=M x(t)-\widehat{d}_{1}(t)-\widehat{F}(t),
$$

where $M$ is the state feedback controller gain to be determined later. Substituting (16) into (7), and it is possible to obtain the augmented system

$$
\begin{aligned}
{\left[\begin{array}{c}
\dot{x}(t) \\
\dot{e}(t)
\end{array}\right]=} & {\left[\begin{array}{cc}
A & B_{1} E \\
0 & W_{1}
\end{array}\right]\left[\begin{array}{l}
x(t) \\
e(t)
\end{array}\right] } \\
& +\left[\begin{array}{cc}
B_{1} M & 0 \\
0 & N B_{1} E
\end{array}\right]\left[\begin{array}{c}
x(t-\Delta(t)) \\
e(t-\Delta(t))
\end{array}\right] \\
& +\left[\begin{array}{ccc}
B_{2} & 0 & 0 \\
0 & N B_{2} & H_{3}
\end{array}\right]\left[\begin{array}{c}
d_{2}(t) \\
d_{2}(t-\Delta(t)) \\
\delta(t)
\end{array}\right] \\
& +\left[\begin{array}{c}
0 \\
H_{1}
\end{array}\right] \dot{F}(t) .
\end{aligned}
$$

Then the reference output equation in the $H_{\infty}$ preference index can be described as

$$
z(t)=\left[\begin{array}{ll}
C_{11} & C_{12}
\end{array}\right]\left[\begin{array}{c}
x(t) \\
e(t)
\end{array}\right]+\left[\begin{array}{ll}
C_{21} & C_{22}
\end{array}\right]\left[\begin{array}{c}
x(t-\Delta(t)) \\
e(t-\Delta(t))
\end{array}\right] .
$$

Denoting $\bar{x}(t)=\left[\begin{array}{ll}x(t) & e(t)\end{array}\right]^{T}$, then (17) and (18) can be described as

$$
\begin{gathered}
\dot{\bar{x}}(t)=A_{0} \bar{x}(t)+A_{d} \bar{x}(t-\Delta(t))+B_{0} \bar{w}_{0}(t)+B_{F} \dot{F}(t), \\
z(t)=C_{1} \bar{x}(t)+C_{2} \bar{x}(t-\Delta(t)),
\end{gathered}
$$

$$
\Theta_{1}=\left[\begin{array}{cc}
\Pi_{11} & \tau A_{0}^{T} S^{-1} A_{d}+C_{1}^{T} C_{2} \\
* & -(1-\bar{d}) Q+\tau A_{d}^{T} S^{-1} A_{d}+C_{2}^{T} C_{2} \\
* & * \\
* & *
\end{array}\right.
$$

where

$$
\begin{aligned}
\Pi_{11}= & \operatorname{sym}\left[P\left(A+A_{d}\right)\right]+Q+\tau A_{0}^{T} S^{-1} A_{0} \\
& +\tau(1-\bar{d})^{-1} P A_{d} S A_{d}^{T} P+C_{1}^{T} C_{1} .
\end{aligned}
$$

Define

$$
x_{d}(t)=x(t-\Delta(t))=x(t)-\int_{t-\Delta(t)}^{t} \xi(s) d s,
$$

where

$$
\xi(s)=A x(s)+A_{d} x_{d}(s)+B_{0} w_{0}(s)+B_{F} w_{F}(s) .
$$

where

$$
\begin{aligned}
& A_{0}=\left[\begin{array}{cc}
A & B_{1} E \\
0 & W_{1}
\end{array}\right], \quad A_{d}=\left[\begin{array}{cc}
B_{1} M & 0 \\
0 & N B_{1} E
\end{array}\right], \\
& B_{0}=\left[\begin{array}{ccc}
B_{2} & 0 & 0 \\
0 & N B_{2} & H_{3}
\end{array}\right], \quad B_{F}=\left[\begin{array}{c}
0 \\
H_{1}
\end{array}\right] \text {, } \\
& \bar{w}_{0}=\left[\begin{array}{lll}
d_{2}(t) & d_{2}(t-\Delta(t)) & \delta(t)
\end{array}\right]^{T}, \\
& C_{1}=\left[\begin{array}{ll}
C_{11} & C_{12}
\end{array}\right], \quad C_{2}=\left[\begin{array}{ll}
C_{21} & C_{22}
\end{array}\right] .
\end{aligned}
$$

At this stage, the objective is to find $K, L$, and $M$ such that system (17) is robustly asymptotically stable and satisfies the generalized $H_{\infty}$ performance. We give the following theorem for the concerned robust fault diagnosis problem.

Lemma 2. For composite system (21), the parameters $\gamma_{1}>0$, $\gamma_{2}>0$, if there exist matrices $P>0, Q>0, S>0$, and $S \leq P^{-1}$, satisfying

$$
\left[\begin{array}{ccccccc}
\Phi_{11} & 0 & P B_{0} & P B_{F} & \tau A_{0}^{T} & \tau P A_{d} & C_{1}^{T} \\
* & -\sigma Q & 0 & 0 & \tau A_{d}^{T} & 0 & C_{2}^{T} \\
* & * & -\gamma_{0}^{2} I & 0 & \tau B_{0}^{T} & 0 & 0 \\
* & * & * & -\gamma_{1}^{2} I & \tau B_{F}^{T} & 0 & 0 \\
* & * & * & * & -\tau S & 0 & 0 \\
* & * & * & * & * & -\tau \sigma P & 0 \\
* & * & * & * & * & * & -I
\end{array}\right]<0
$$

where $\Phi_{11}=\operatorname{sym}\left[P\left(A_{0}+A_{d}\right)\right]+Q, \sigma=1-\bar{d}$, then the composite system (21) is robustly asymptotically stable and satisfies $\|z(t)\|_{2}^{2}<\gamma_{0}^{2}\left\|w_{0}(t)\right\|_{2}^{2}+\gamma_{1}^{2}\left\|w_{F}(t)\right\|_{2}^{2}$.

Proof. First, we prove the stability of the control system, according to the Schur complement formula and $S \leq P^{-1}$, the linear matrix inequalities (21) can be transformed into

$$
\left.\begin{array}{cc}
P B_{0}+\tau A_{0}^{T} S^{-1} B_{0} & P B_{F}+\tau A_{0}^{T} S^{-1} B_{F} \\
\tau A_{d}^{T} S^{-1} B_{0} & \tau A_{D}^{T} S^{-1} B_{F} \\
-\gamma_{0}^{2} I+\tau B_{0}^{T} S^{-1} B_{0} & \tau B_{0}^{T} S^{-1} B_{F} \\
* & -\gamma_{1}^{2} I+\tau B_{F}^{T} S^{-1} B_{F}
\end{array}\right]<0,
$$

A Lyapunov function candidate for system (21) is chosen as

$$
\begin{aligned}
V(x, t)= & x^{T}(t) P x(t)+\int_{t-\Delta(t)}^{t} x^{T}(s) Q x(s) d s \\
& +\int_{-\Delta(t)}^{0} \int_{t+\beta}^{t} \xi^{T}(s) S^{-1} \xi(s) d s d \beta .
\end{aligned}
$$

It can be shown that

$$
\begin{aligned}
& \dot{V}(x(t), t) \\
&=\dot{x}^{T}(t) P x(t)+x^{T}(t) P \dot{x}(t) \\
&+x^{T}(t) Q x(t)-[(1-\dot{\Delta}(t))] x_{d}^{T}(t) Q x_{d}(t) \\
&+\dot{\Delta}(t) \int_{t-\Delta(t)}^{t} \xi^{T}(s) S^{-1} \xi(s) d s+\Delta(t) \xi^{T}(t) S^{-1} \xi(t)
\end{aligned}
$$




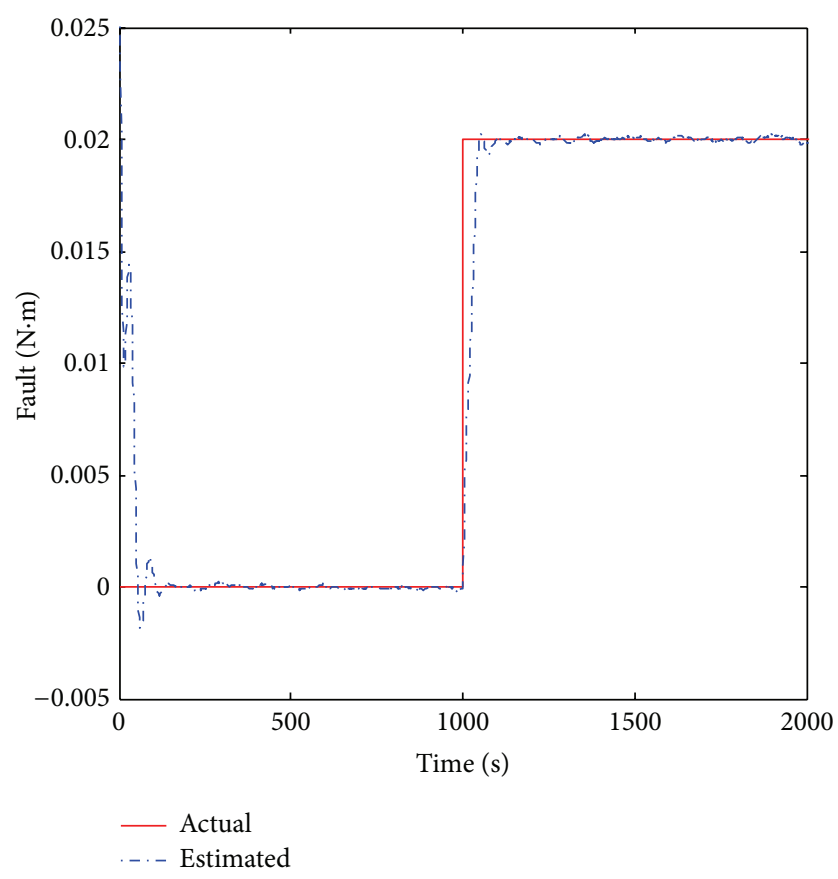

FIGURE 1: Curves of stuck fault and its estimation.

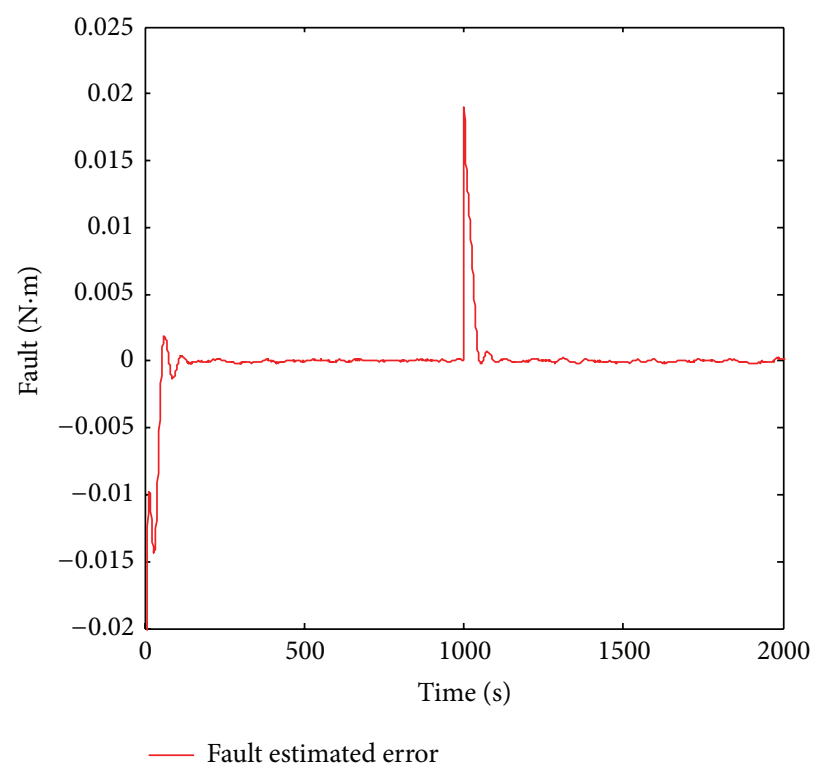

FIgURE 2: Curves of the estimation error of stuck faults.

$$
\begin{aligned}
& -\int_{-\Delta(t)}^{0} \xi^{T}(t+\beta) S^{-1} \xi(t+\beta) d \beta \\
\leq & \alpha^{T}(t)\left(\Theta_{1}-\Theta_{2}\right) \alpha(t),
\end{aligned}
$$

where

$$
\alpha(t)=\left[\begin{array}{c}
x(t) \\
x_{d}(t) \\
w_{0}(t) \\
w_{F}(t)
\end{array}\right],
$$

$$
\Theta_{2}=\left[\begin{array}{cccc}
C_{1}^{T} C_{1} & C_{1}^{T} C_{2} & 0 & 0 \\
* & C_{2}^{T} C_{2} & 0 & 0 \\
* & * & -\gamma_{0}^{2} I & 0 \\
* & * & * & -\gamma_{1}^{2} I
\end{array}\right] .
$$

By using Schur complement formula that $\Theta_{1}<0$ leads to $\Theta_{1}-\Theta_{2}<0$, it can be obtained that $\dot{V}(x(t), t) \leq 0$, 
which implies that the system (17) is robustly asymptotically stable.

The next step is to prove the robustness of the system. Consider an auxiliary function as the performance index

$$
\begin{aligned}
& J(x(t)) \\
& \quad=V(x(t), t)+\int_{0}^{t}\|z(s)\|_{2}^{2}-\gamma_{0}^{2}\left\|w_{0}(s)\right\|_{2}^{2}-\gamma_{1}^{2}\left\|w_{F}(s)\right\|_{2}^{2} d s,
\end{aligned}
$$

which satisfies the zero initial condition. Similar to the proof of the stability, from (21) we have

$$
\begin{aligned}
\dot{J}(x(t) & =\dot{V}(x(t), t)+\|z(t)\|_{2}^{2}-\gamma_{0}^{2}\left\|w_{0}(t)\right\|_{2}^{2}-\gamma_{1}^{2}\left\|w_{F}(t)\right\|_{2}^{2} \\
& \leq \alpha^{T}(t)\left(\Theta_{1}-\Theta_{2}\right) \alpha(t)<0 .
\end{aligned}
$$

Then, we can draw a conclusion that $J(x(t))<0$ under the zero initial condition which further leads to $\|z(t)\|_{2}^{2}<$ $\gamma_{0}^{2}\left\|w_{0}(t)\right\|_{2}^{2}+\gamma_{1}^{2}\left\|w_{F}(t)\right\|_{2}^{2}$. The proof is completed.

Theorem 3. For composite system (18), the parameters $\gamma_{0}>0$, $\gamma_{1}>0, \gamma_{2}>0$, and $\gamma_{3}>0$, if there exist matrices $X>0$, $Y>0, P_{2}>0, Q_{2}>0, T>0, S_{1}>0$, and $S_{1}<X, T<P_{2}$, satisfying

$$
\left[\begin{array}{ccccccccccccc}
\phi_{11} & B_{1} E & 0 & 0 & B_{2} & 0 & 0 & 0 & \tau X A^{T} & 0 & \tau B_{1} R_{1} & 0 & X C_{11}^{T} \\
* & \phi_{22} & 0 & 0 & 0 & R_{2} B_{2} & P_{2} H_{3} & P_{2} H_{1} & \tau E^{T} B_{1}^{T} & \tau W_{1}^{T} P_{2} & 0 & R_{2} B_{1} E & C_{12}^{T} \\
* & * & -\sigma Y & 0 & 0 & 0 & 0 & 0 & \tau R_{1}^{T} B_{1}^{T} & 0 & 0 & 0 & X C_{21}^{T} \\
* & * & * & -\sigma Q_{2} & 0 & 0 & 0 & 0 & 0 & \tau E^{T} B_{1}^{T} R_{2}^{T} & 0 & 0 & C_{22}^{T} \\
* & * & * & * & -\gamma_{0}^{2} I & 0 & 0 & 0 & \tau B_{2}^{T} & 0 & 0 & 0 & 0 \\
* & * & * & * & * & -\gamma_{1}^{2} I & 0 & 0 & 0 & \tau B_{2}^{T} R_{2}^{T} & 0 & 0 & 0 \\
* & * & * & * & * & * & -\gamma_{2}^{2} I & 0 & 0 & \tau H_{3}^{T} P_{2} & 0 & 0 & 0 \\
* & * & * & * & * & * & * & -\gamma_{3}^{2} I & 0 & \tau H_{1}^{T} P_{2} & 0 & 0 & 0 \\
* & * & * & * & * & * & * & * & -\tau S_{1} & 0 & 0 & 0 & 0 \\
* & * & * & * & * & * & * & * & * & -\tau T & 0 & 0 & 0 \\
* & * & * & * & * & * & * & * & * & * & -\tau \sigma X & 0 & 0 \\
* & * & * & * & * & * & * & * & * & * & * & -\tau \sigma P_{2} & 0 \\
* & * & * & * & * & * & * & * & * & * & * & * & -I
\end{array}\right]
$$

where $\phi_{11}=\operatorname{sym}\left(A X+B_{1} R_{1}\right)+Y, \phi_{22}=\operatorname{sym}\left(P_{2} W_{1}+\right.$ $\left.R_{2} B_{1} E\right)+Q_{2}$, then the composite system (18) with controller gain $M=R_{1} X^{-1}$ and observer gain $\left[\begin{array}{c}L \\ K\end{array}\right]=P_{2}^{-1} R_{2}$ is robustly asymptotically stable and satisfies

$$
\begin{aligned}
& \|z(t)\|_{2}^{2} \\
& \qquad \gamma_{0}^{2}\left\|d_{2}(t)\right\|_{2}^{2}+\gamma_{1}^{2}\left\|d_{2}(t-\Delta(t))\right\|_{2}^{2}+\gamma_{2}^{2}\|\delta(t)\|_{2}^{2} \\
& \quad+\gamma_{3}^{2}\|\dot{F}(t)\|_{2}^{2} .
\end{aligned}
$$

Proof. According to the system (18), denoting

$$
P=\left[\begin{array}{cc}
P_{1} & 0 \\
0 & P_{2}
\end{array}\right], \quad Q=\left[\begin{array}{cc}
Q_{1} & 0 \\
0 & Q_{2}
\end{array}\right], \quad S=\left[\begin{array}{cc}
S_{1} & 0 \\
0 & S_{2}
\end{array}\right]
$$

Substituting the related matrix into Lemma 2, we can get

$$
\left[\begin{array}{ccccccccccccc}
\varphi_{11} & P_{1} B_{1} E & 0 & 0 & P_{1} B_{2} & 0 & 0 & 0 & \tau A^{T} & 0 & \tau P_{1} B_{1} N & 0 & C_{11}^{T} \\
* & \varphi_{22} & 0 & 0 & 0 & R_{2} B_{2} & P_{2} H_{3} & P_{2} H_{1} & \tau E^{T} B_{1}^{T} & \tau W_{1}^{T} & 0 & R_{2} B_{1} E & C_{12}^{T} \\
* & * & -\sigma Y & 0 & 0 & 0 & 0 & 0 & \tau M^{T} B_{1}^{T} & 0 & 0 & 0 & C_{21}^{T} \\
* & * & * & -\sigma Q_{2} & 0 & 0 & 0 & 0 & 0 & \tau E^{T} B_{1}^{T} N^{T} & 0 & 0 & C_{22}^{T} \\
* & * & * & * & -\gamma_{0}^{2} I & 0 & 0 & 0 & \tau B_{2}^{T} & 0 & 0 & 0 & 0 \\
* & * & * & * & * & -\gamma_{1}^{2} I & 0 & 0 & 0 & \tau B_{2}^{T} N^{T} & 0 & 0 & 0 \\
* & * & * & * & * & * & -\gamma_{2}^{2} I & 0 & 0 & \tau H_{3}^{T} & 0 & 0 & 0 \\
* & * & * & * & * & * & * & -\gamma_{3}^{2} I & 0 & \tau H_{1}^{T} & 0 & 0 & 0 \\
* & * & * & * & * & * & * & * & -\tau S_{1} & 0 & 0 & 0 & 0 \\
* & * & * & * & * & * & * & * & * & -\tau S_{2} & 0 & 0 & 0 \\
* & * & * & * & * & * & * & * & * & * & -\tau \sigma P_{1} & 0 & 0 \\
* & * & * & * & * & * & * & * & * & * & * & -\tau \sigma P_{2} & 0 \\
* & * & * & * & * & * & * & * & * & * & * & * & -I
\end{array}\right]
$$




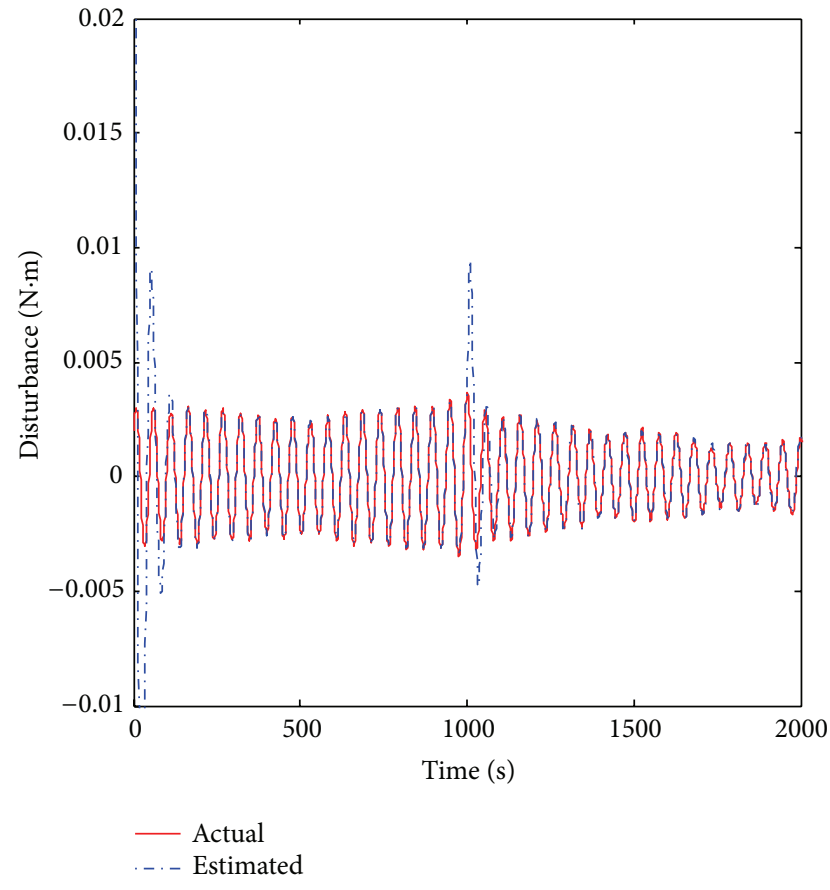

(a) Time responses of vibration and vibration observed

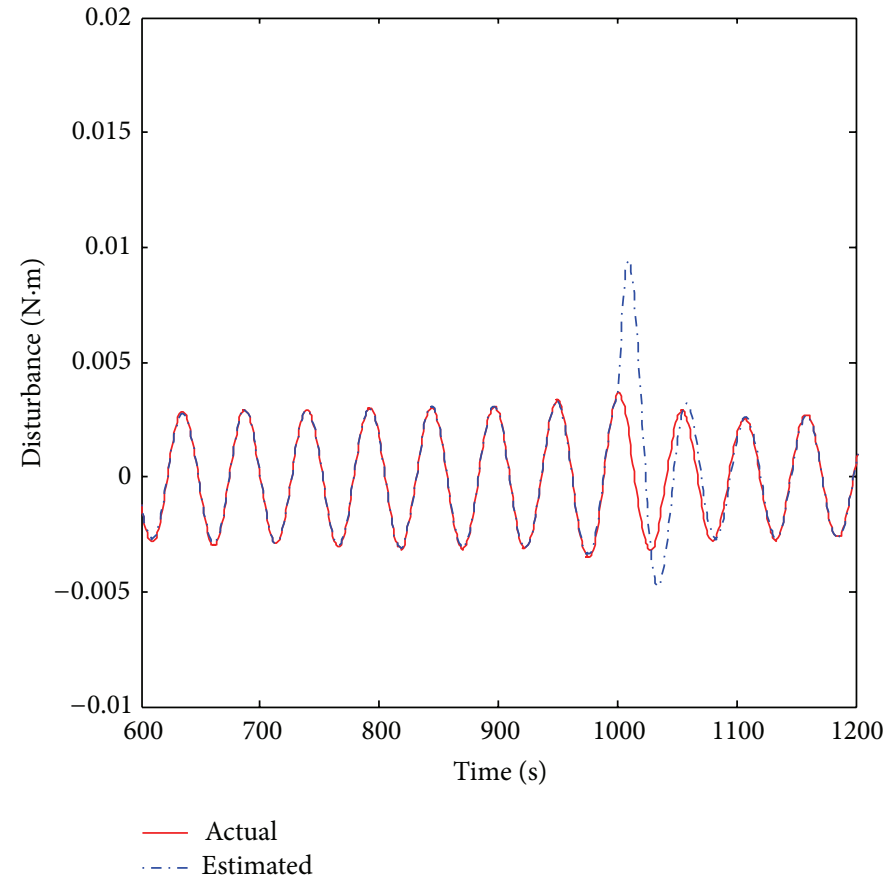

(b) Partial amplification of (a)

FIgURE 3: Curves of vibration estimation of disturbance observer.

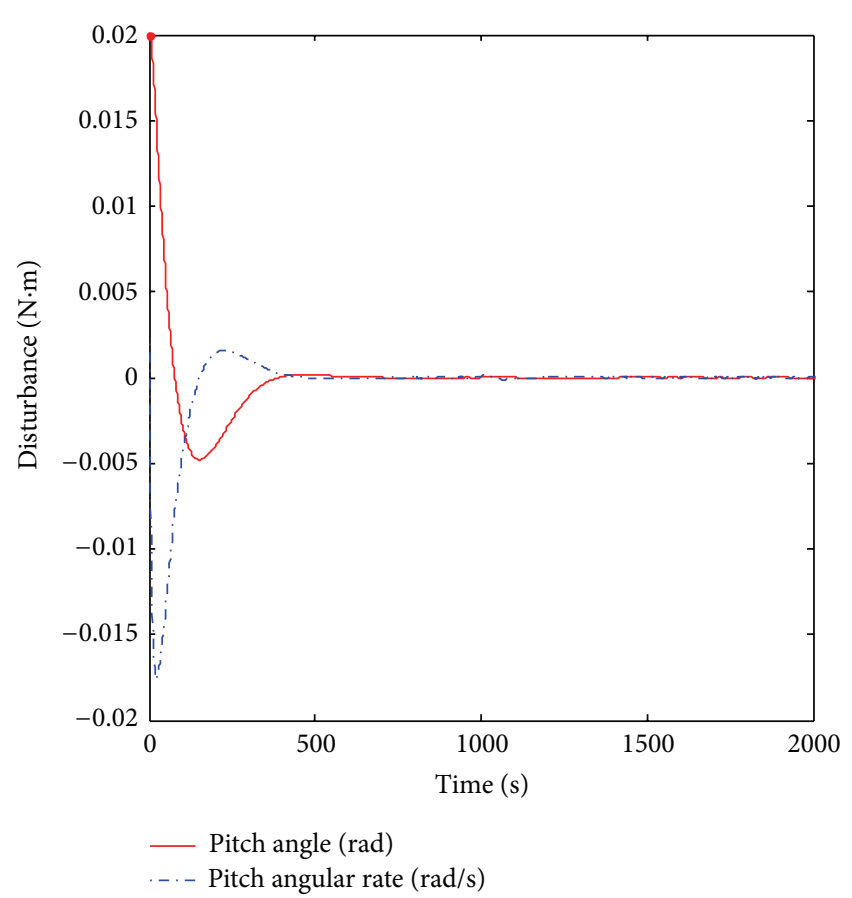

(a) Time responses of attitude angle and attitude angular velocity

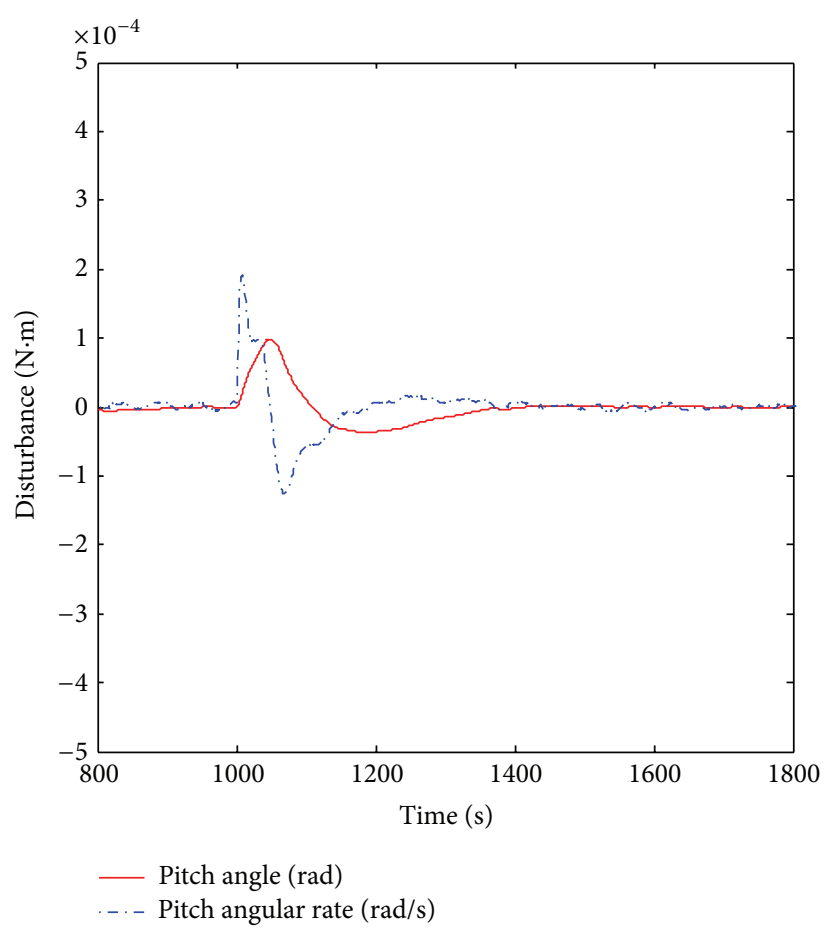

(b) Partial amplification of (a)

FIGURE 4: Time responses of attitude angle and attitude angular velocity. 
where $\varphi_{11}=\operatorname{sym}\left(P_{1} A+B_{1} M\right)+Q_{1}, \varphi_{22}=\operatorname{sym}\left(P_{2} W_{1}+\right.$ $\left.R_{2} B_{1} E\right)+Q_{2}$, premultiply and postmultiply $\operatorname{diag}\{X, I, X, I, I$, $\left.I, I, I, I, P_{2}, X, I, I\right\}$ simultaneously to the left and right sides of (34), and then defining $X=P_{1}^{-1}, R_{1}=M P_{1}^{-1}=M X$, $R_{2}=P_{2} N, Y=X Q_{1} X$, and $T=P_{2} S_{2} P_{2}$ then (31) is obtained. Furthermore, $S_{1} \leq P_{1}^{-1}=X$ and $S_{2} \leq P_{2}^{-1}$ are equivalent to $T \leq P_{2}$. So, the composite system (18) with controller gain $M=R_{1} X^{-1}$ and $N=P_{2}^{-1} R_{2}$ is robustly asymptotically stable and satisfies $\|z(t)\|_{2}^{2}<\gamma_{0}^{2}\left\|d_{2}(t)\right\|_{2}^{2}+\gamma_{1}^{2}\left\|d_{2}(t-\Delta(t))\right\|_{2}^{2}+$ $\gamma_{2}^{2}\|\delta(t)\|_{2}^{2}+\gamma_{3}^{2}\|\dot{F}(t)\|_{2}^{2}$.

\section{Simulation Example}

In order to demonstrate the effectiveness of the proposed control algorithm, numerical simulations will be performed in this section. The composite controller will be applied for the attitude control of a microsatellite with unknown input delay, reaction wheel's faults, and vibration disturbance torque from reaction wheel simultaneously.

In this paper, we only consider the attitude in the pitch channel. Select the upper bound $\tau=30 \mathrm{~ms}$, and $\bar{d}=0.1$. The microsatellite is supposed to move in a circular orbit with the altitude of $900 \mathrm{~km}$; then the orbit rate $n=0.0011 \mathrm{rad} / \mathrm{s}$. $J=6.14 \mathrm{~kg} \cdot \mathrm{m}^{2}$ is the nominal principal moment of inertia of pitch axis. The initial pitch attitudes of the microsatellite are $\theta=0.02 \mathrm{rad}$ and $\dot{\theta}=0.002 \mathrm{rad} / \mathrm{s}$. Periodic disturbances $d_{1}(t)$ caused by reaction wheel are described by $(10)$ with $W=$ $\left[\begin{array}{cc}0 & 6 \\ -6 & 0\end{array}\right], V=\left[\begin{array}{cc}5 & 0\end{array}\right]$. We select $\delta(t)$ as the random signal with upper 2-norm bound 1. $d_{2}(t)$ can also be considered as the random signal with bounded upper 2-norm. For the reference output, it is denoted that $C_{11}=\left[\begin{array}{lll}0.2 & 0.2 & 0.2\end{array}\right], C_{12}=$ $\left[\begin{array}{lll}0.8 & 0.2 & 0\end{array}\right], C_{21}=\left[\begin{array}{lll}0 & 0.2 & 0.8\end{array}\right]$, and $C_{22}=\left[\begin{array}{lll}0.8 & 0.2 & 0\end{array}\right]$. For $\sigma=0.9$ and $\gamma_{0}=1, \gamma_{1}=2, \gamma_{2}=0.5$, and $\gamma_{3}=1.2$. It can be solved via LMI related to (17) that the gain of fault diagnosis observer (11) is

$$
K=\left[\begin{array}{lll}
0 & 0 & -20.4023
\end{array}\right]
$$

the gain of disturbance observer (12) is

$$
L=\left[\begin{array}{lll}
0 & 0 & -4.3713 \\
0 & 0 & -2.3608
\end{array}\right],
$$

and the gain of state feedback controller is

$$
M=\left[\begin{array}{lll}
-17.2607 & -37.7208 & -38.7539
\end{array}\right] .
$$

The stuck fault of reaction wheel is supposed to occur at the 1000th second as $F=0.02 \mathrm{~N} \cdot \mathrm{m}$. The estimation of stuck faults is demonstrated in Figure 1, where the solid line represents the real fault signal and the dash-dot line stands for their estimation. Fault estimation error is shown in Figure 2. From Figures 1 and 2, it can be seen that the proposed fault diagnosis method has respectable estimation ability.

When the disturbance observer is constructed based on (12), Figure 3(a) shows the actual value, and estimated value of the disturbance caused by the reaction wheel. Figure 3(b) is obtained by partially amplifying Figure 3(a). From both figures, we can see that the main disturbance can be estimated and rejected accurately by the proposed disturbance observer.

Figures 4(a) and 4(b) show that the composite controller is capable of compensating the effect of stuck faults and vibration disturbance torque from reaction wheel actively and can improve the reliability and stability of the microsatellite in the presence of the model uncertainty and space environmental disturbances.

\section{Conclusion}

In this paper, the fault diagnosis problem is addressed for microsatellite with unknown input delay. The following features of the proposed algorithm are compared with the previous results. Firstly, a uniform fault diagnosis observer is constructed for the attitude control systems. Secondly, the disturbances considered in this paper are assumed to include vibration disturbance torque from reaction wheel and norm bounded uncertain disturbance. A disturbance observer is constructed to estimate the vibration disturbance. Thirdly, a new composite controller is designed which can attenuate the influence of the norm bounded disturbances and the estimation errors and correspondingly guarantee the robust stability against other disturbances. Finally, simulations for a microsatellite are given to show the efficiency of the proposed approach. Although numerical simulations have shown that enhanced robustness can be achieved by using the proposed method, more general theoretical research and experimental simulations need to be carried out in the future to ensure the reliability and stability of attitude control systems for microsatellite.

\section{Conflict of Interests}

There is no conflict of interests regarding the publication of this paper.

\section{Acknowledgments}

This work was supported by the National 973 Program (Grant no. 2012CB720003), and the National Natural Science Foundation of China (NSFC) (Grants no. 60925012, 61127007, 91016004, and 61121003).

\section{References}

[1] M. Tafazoli, "A study of on-orbit spacecraft failures," Acta Astronautica, vol. 64, no. 2-3, pp. 195-205, 2009.

[2] J.-F. Castet and J. H. Saleh, "Beyond reliability, multi-state failure analysis of satellite subsystems: a statistical approach," Reliability Engineering and System Safety, vol. 95, no. 4, pp. 311322, 2010.

[3] W.-Y. Zhou, G. S. Aglietti, and Z. Zhang, "Modelling and testing of a soft suspension design for a reaction/momentum wheel assembly," Journal of Sound and Vibration, vol. 330, no. 18-19, pp. 4596-4610, 2011.

[4] S. Taniwaki and Y. Ohkami, "Experimental and numerical analysis of reaction wheel disturbances," JSME International Journal C, vol. 46, no. 2, pp. 519-526, 2003. 
[5] H.-S. Oh and D.-I. Cheon, "Precision measurements of reaction wheel disturbances with frequency compensation process," Journal of Mechanical Science and Technology, vol. 19, no. 1, pp. 136-143, 2005.

[6] R. A. Masterson, D. W. Miller, and R. L. Grogan, "Development and validation of reaction wheel disturbance models: empirical model," Journal of Sound and Vibration, vol. 249, no. 3, pp. 575598, 2002.

[7] C.-Y. Kao and A. Rantzer, "Stability analysis of systems with uncertain time-varying delays," Automatica, vol. 43, no. 6, pp. 959-970, 2007.

[8] J. Lam, H. Gao, and C. Wang, "Stability analysis for continuous systems with two additive time-varying delay components," Systems and Control Letters, vol. 56, no. 1, pp. 16-24, 2007.

[9] M. Blanke, M. Kinnaert, M. Lunze et al., Diagnosis and Fault Tolerant Control, Springer, New York, NY, USA, 2003.

[10] M. Zhong, H. Ye, S. X. Ding, and G. Wang, "Observer-based fast rate fault detection for a class of multirate sampled-data systems," IEEE Transactions on Automatic Control, vol. 52, no. 3, pp. 520-525, 2007.

[11] W. Wang, D. H. Zhou, and Z. Li, "Robust state estimation and fault diagnosis for uncertain hybrid systems," Nonlinear Analysis: Theory, Methods and Applications, vol. 65, no. 12, pp. 2193-2215, 2006

[12] L. Guo, Y.-M. Zhang, H. Wang, and J.-C. Fang, "Observer-based optimal fault detection and diagnosis using conditional probability distributions," IEEE Transactions on Signal Processing, vol. 54, no. 10, pp. 3712-3719, 2006.

[13] Z. Gao and S. X. Ding, "Actuator fault robust estimation and fault-tolerant control for a class of nonlinear descriptor systems," Automatica, vol. 43, no. 5, pp. 912-920, 2007.

[14] J. Jin, S. Ko, and C.-K. Ryoo, "Fault tolerant control for satellites with four reaction wheels," Control Engineering Practice, vol. 16, no. 10, pp. 1250-1258, 2008.

[15] N. Meskin and K. Khorasani, "Fault detection and isolation in a redundant reaction wheels configuration of a satellite," in Proceedings of the IEEE International Conference on Systems, Man, and Cybernetics (SMC '07), pp. 3153-3158, October 2007.

[16] N. Tudoroiu, E. Sobhani-Tehrani, and K. Khorasani, "Interactive bank of unscented Kalman filters for fault detection and isolation in reaction wheel actuators of satellite attitude control system," in Proceedings of the 32nd Annual Conference on IEEE Industrial Electronics (IECON '06), pp. 264-269, November 2006.

[17] D. Hu, A. Sarosh, and Y.-F. Dong, "A novel KFCM based fault diagnosis method for unknown faults in satellite reaction wheels," ISA Transactions, vol. 51, no. 2, pp. 309-316, 2012.

[18] Q. Hu, "Robust adaptive sliding-mode fault-tolerant control with L2-gain performance for flexible spacecraft using redundant reaction wheels," IET Control Theory and Applications, vol. 4, no. 6, pp. 1055-1070, 2010.

[19] Q. Hu, Y. Zhang, X. Huo, and B. Xiao, "Adaptive integral-type sliding mode control for spacecraft attitude maneuvering under actuator stuck failures," Chinese Journal of Aeronautics, vol. 24, no. 1, pp. 32-45, 2011.

[20] A.-M. Zou and K. D. Kumar, "Adaptive fuzzy fault-tolerant attitude control of spacecraft," Control Engineering Practice, vol. 19, no. 1, pp. 10-21, 2011.

[21] L. Guo and W.-H. Chen, "Disturbance attenuation and rejection for systems with nonlinearity via DOBC approach," International Journal of Robust and Nonlinear Control, vol. 15, no. 3, pp. 109-125, 2005.
[22] L. Guo, C. B. Feng, and W. H. Chen, "A survey of disturbanceobserver-based control for dynamic nonlinear system," Dynamics of Continuous Discrete and Impulsive System B, vol. 13E, pp. 79-84, 2006.

[23] S. Cao, L. Guo, and X. Wen, "Fault tolerant control with disturbance rejection and attenuation performance for systems with multiple disturbances," Asian Journal of Control, vol. 13, no. 6, pp. 1056-1064, 2011.

[24] L. Guo, "Composite Hierarchical Anti-Disturbance Control (CHADC) for systems with multiple disturbances: Survey and overview," in Proceedings of the 30th Chinese Control Conference (CCC '11), pp. 6193-6198, July 2011.

[25] H. Liu, L. Guo, and Y. Zhang, "An anti-disturbance PD control scheme for attitude control and stabilization of flexible spacecrafts," Nonlinear Dynamics, vol. 67, no. 3, pp. 2081-2088, 2012.

[26] H. Liu, L. Guo, and Y. Zhang, "Composite attitude control for flexible spacecraft with simultaneous disturbance attenuation and rejection performance," Proceedings of the Institution of Mechanical Engineers I, vol. 226, no. 2, pp. 154-161, 2012.

[27] J. Z. Qiao, L. Guo, Y. J. Lei et al., "Subtle anti-disturbance tolerant control of attitude control systems for microsatellites," Scientia Sinica Informationis, vol. 42, pp. 1327-1337, 2012. 


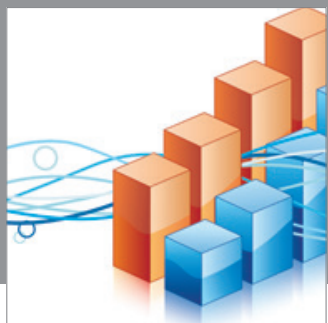

Advances in

Operations Research

mansans

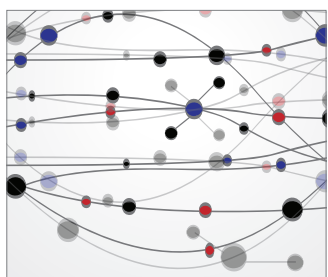

The Scientific World Journal
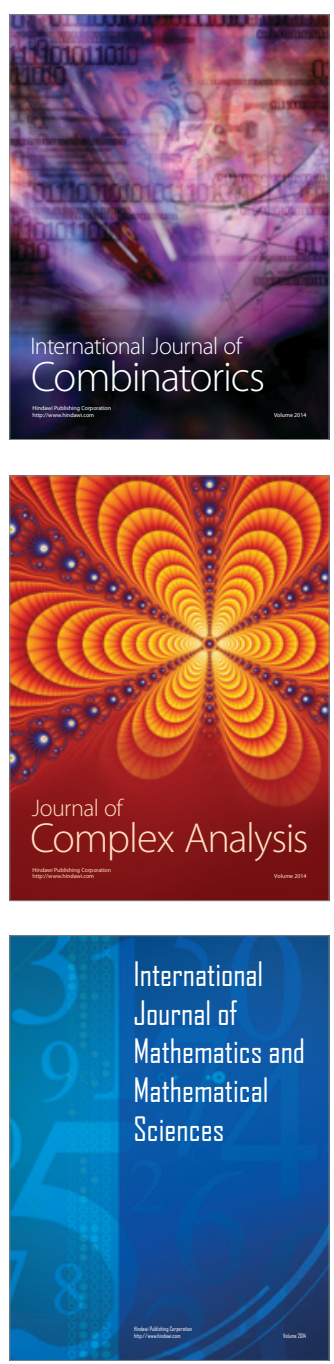
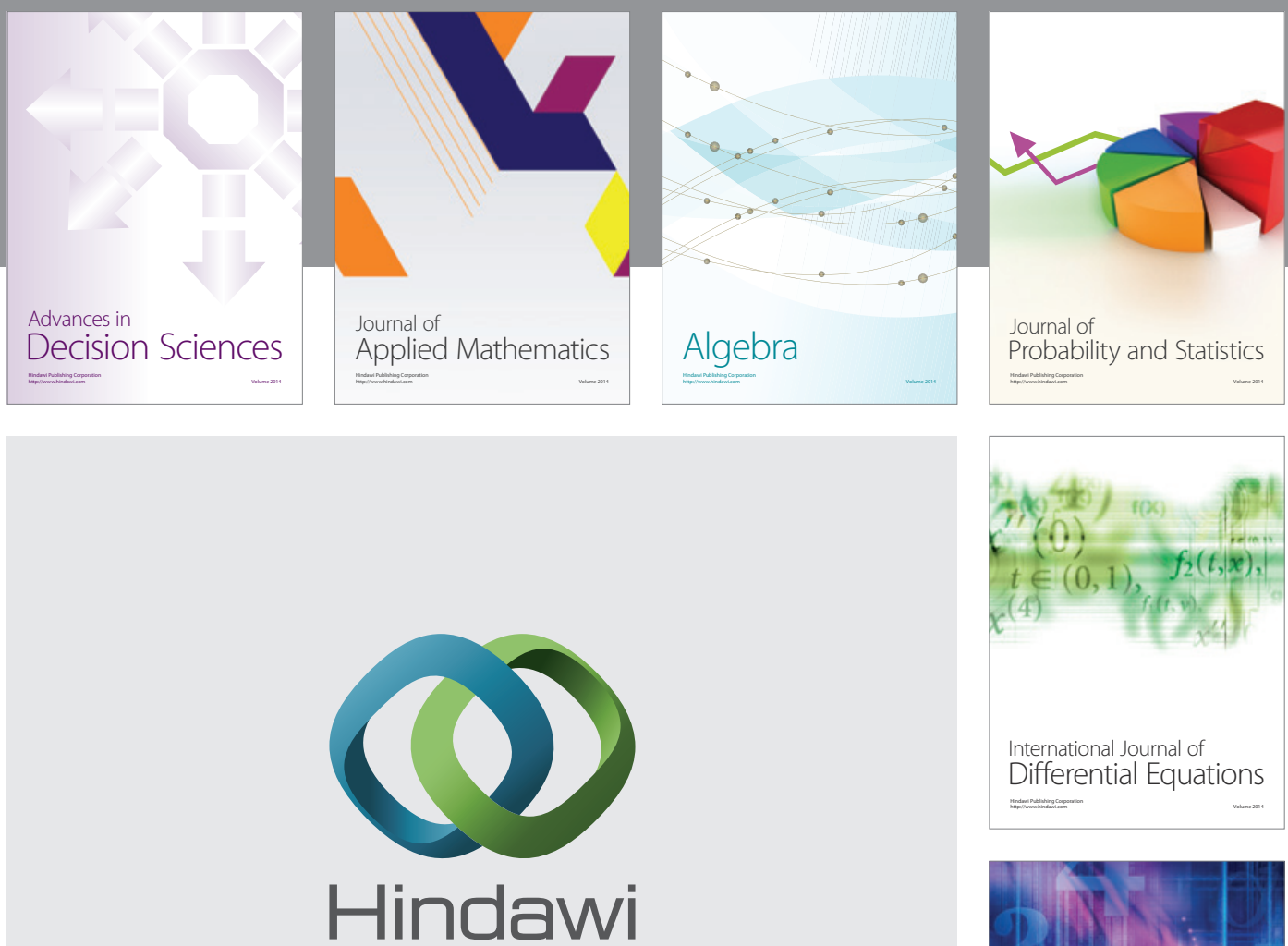

Submit your manuscripts at http://www.hindawi.com
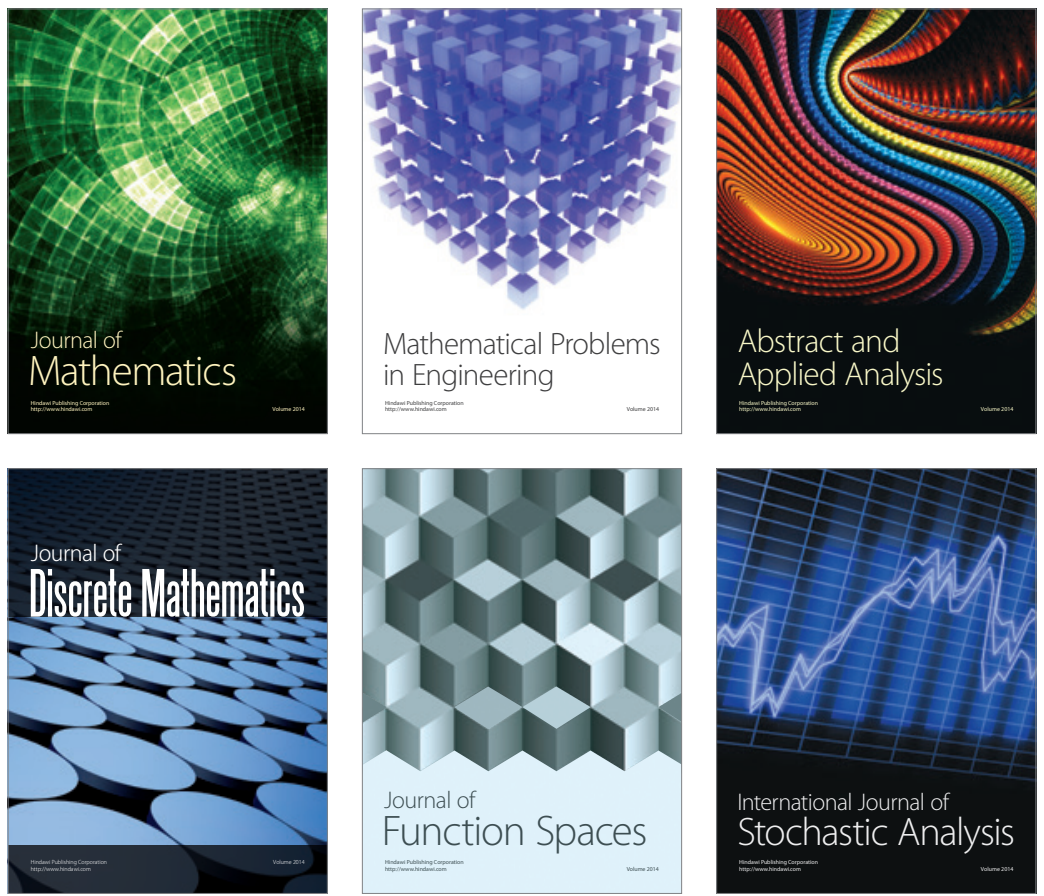

Journal of

Function Spaces

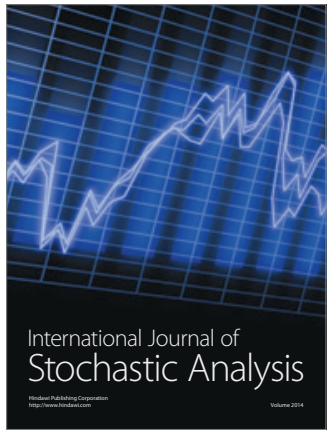

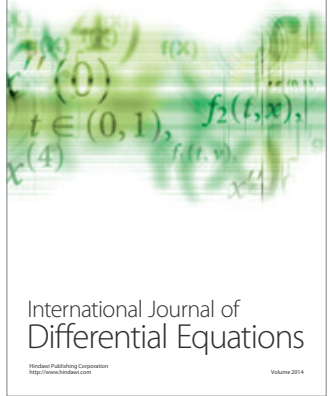
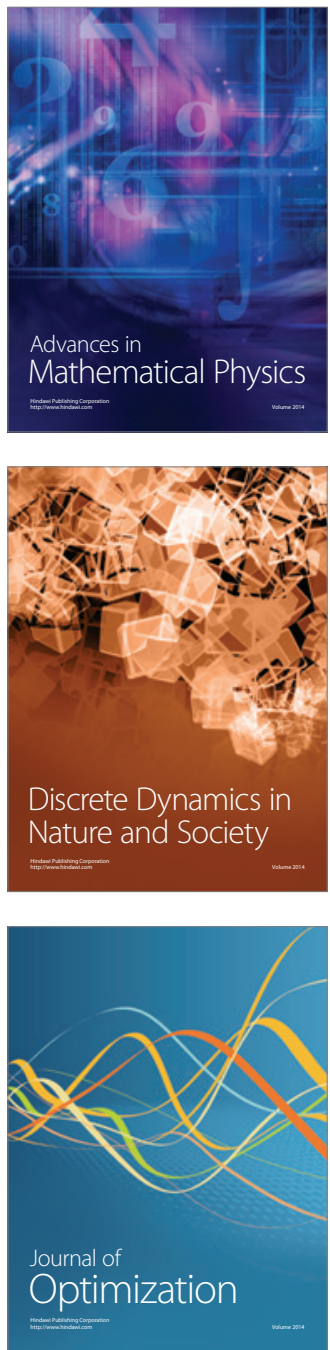